Tohoku J. Exp. Med., 2008, 214, 359-363

\title{
Successful Treatment of Carcinomatous Meningitis with Gefitinib in a Patient with Lung Adenocarcinoma Harboring a Mutated EGF Receptor Gene
}

\author{
Tatsuro Fukuhara, ${ }^{1}$ Yasuo Saijo, ${ }^{2}$ Tomohiro Sakakibara, ${ }^{1}$ Akira Inoue, ${ }^{1}$ \\ NaOto Morikawa, ${ }^{1}$ Masayuki Kanamori, ${ }^{3}$ IChiro Nakashima ${ }^{4}$ and \\ TOSHIHIRO NUKIWA ${ }^{1}$ \\ ${ }^{1}$ Department of Respiratory Oncology and Molecular Medicine, Institute of Development, Aging \\ and Cancer, Tohoku University, Sendai, Japan \\ ${ }^{2}$ Department of Medical Oncology, Hirosaki University Graduate School of Medicine, Hirosaki, \\ Japan \\ ${ }^{3}$ Department of Neurosurgery, Tohoku University Graduate School of Medicine, Sendai, Japan \\ ${ }^{4}$ Department of Neurology, Tohoku University Graduate School of Medicine, Sendai, Japan
}

\begin{abstract}
Carcinomatous meningitis is a severe complication of lung cancer. Although treatment with gefitinib, a tyrosine kinase inhibitor of epidermal growth factor (EGF) receptor, has been reported to be highly effective against lung cancers harboring a mutated EGF gene, its effect against carcinomatous meningitis is unknown. Here, we report successful treatment of carcinomatous meningitis with gefitinib in a lung cancer patient suffered from meningeal metastasis. A 62-year-old, non-smoking, Japanese male was admitted for headache, failing vision, and temporary loss of consciousness and was subsequently diagnosed with stage IV lung adenocarcinoma and carcinomatous meningitis. A tumor sample revealed the in-frame deletion of codons 746 to 750 (E746 to A750) in exon 19 of the EGF gene, which leads to constitutive activation of the tyrosine kinase domain and high-affinity binding of gefitinib. The patient's performance status was poor owing to progression of the meningitis and elevated cerebrospinal fluid (CSF) pressure. Combined treatment with gefitinib (250 mg/day) and whole-brain irradiation (36 Gray total) proved to be effective. It is noteworthy that the level of gefitinib in the CSF was less than $1 \%$ of the serum level (serum: $117 \mathrm{nM}$ before drug re-administration and $132 \mathrm{nM} 2 \mathrm{hrs}$ later; CSF: $0.9 \mathrm{nM}$ both before and $2 \mathrm{hrs}$ after drug re-administration). Gefitinib treatment should be considered for patients with carcinomatous meningitis and lung adenocarcinoma harboring a mutated EGF gene. - carcinomatous meningitis; EGF; EGF-TKI; gefitinib; lung cancer.
\end{abstract}

Tohoku J. Exp. Med., 2008, 214 (4), 359-363.

(C) 2008 Tohoku University Medical Press

Metastases to the central nervous system, which are often observed in cases of non-small cell lung cancer (NSCLC), result in poor perfor- mance status of the patient. Although brain metastases can be controlled by whole-brain irradiation (WBI) or $\gamma$-knife irradiation, leptomenin-

Received December 10, 2007; revision accepted for publication March 1, 2008.

Correspondence: Tatsuro Fukuhara, M.D., Ph.D., Department of Respiratory Oncology and Molecular Medicine, Institute of Development, Aging and Cancer, Tohoku University, 4-1, Seiryo-machi, Aoba-ku, Sendai, 980-8575, Japan.

e-mail: t-fuku@idac.tohoku.ac.jp 
geal metastases cannot be treated effectively using current treatment methods.

Gefitinib (Iressa; AstraZeneca, Osaka) is an orally active inhibitor of epidermal growth factor (EGF) receptor-tyrosine kinase. Gefitinib has significant anti-tumor activity against NSCLC, with a response rate of $10-30 \%$, and several recent reports have shown that gefitinib treatment is effective against brain metastases and carcinomatous meningitis (Namba et al. 2004; Kim et al. 2005; Sakai et al. 2006; Wu et al. 2007). Here, we present a case of meningeal metastasis successfully treated with gefitinib and WBI, and we report the patient's cerebrospinal fluid (CSF) and serum levels of gefitinib.

\section{Clinical Findings}

A 62-year-old, non-smoking, Japanese male presented with nausea and neck pain of unknown etiology 3 months prior to admission. On 20 October 2006, he experienced progressive vision loss and a temporary loss of consciousness.
Gadolinium-enhanced T1-weighted imaging revealed high-intensity signals in the opercular parts of the temporal lobe (Fig. 1A), and funduscopic examination showed evidence of papilloedema; thus, carcinomatous meningitis was suspected. Repeated cytological examination of the patient's CSF indicated the presence of Class IV cells, which raised the possibility of adenocarcinoma. Screening by chest X-ray and whole-body $\mathrm{CT}$ revealed the presence of a tumor at a hilus in the right lung (Fig. 1B, C). Cytological examination of the tumor verified the existence of a lung adenocarcinoma. Thus, the patient's diagnosis was stage IV lung cancer with carcinomatous meningitis. The in-frame deletion of codons 746 to 750 (E746 to A750) in exon 19 of EGF was detected using a peptide nucleic acid-locked nucleic acid (PNA-LNA) PCR clamp (Nagai et al. 2005). During the diagnostic period, the patient's headache became severe, and a lumbar puncture revealed high intracranial pressure, exceeding $1,000 \mathrm{~mm} \mathrm{H}_{2} \mathrm{O}$. Continuous drainage of CSF
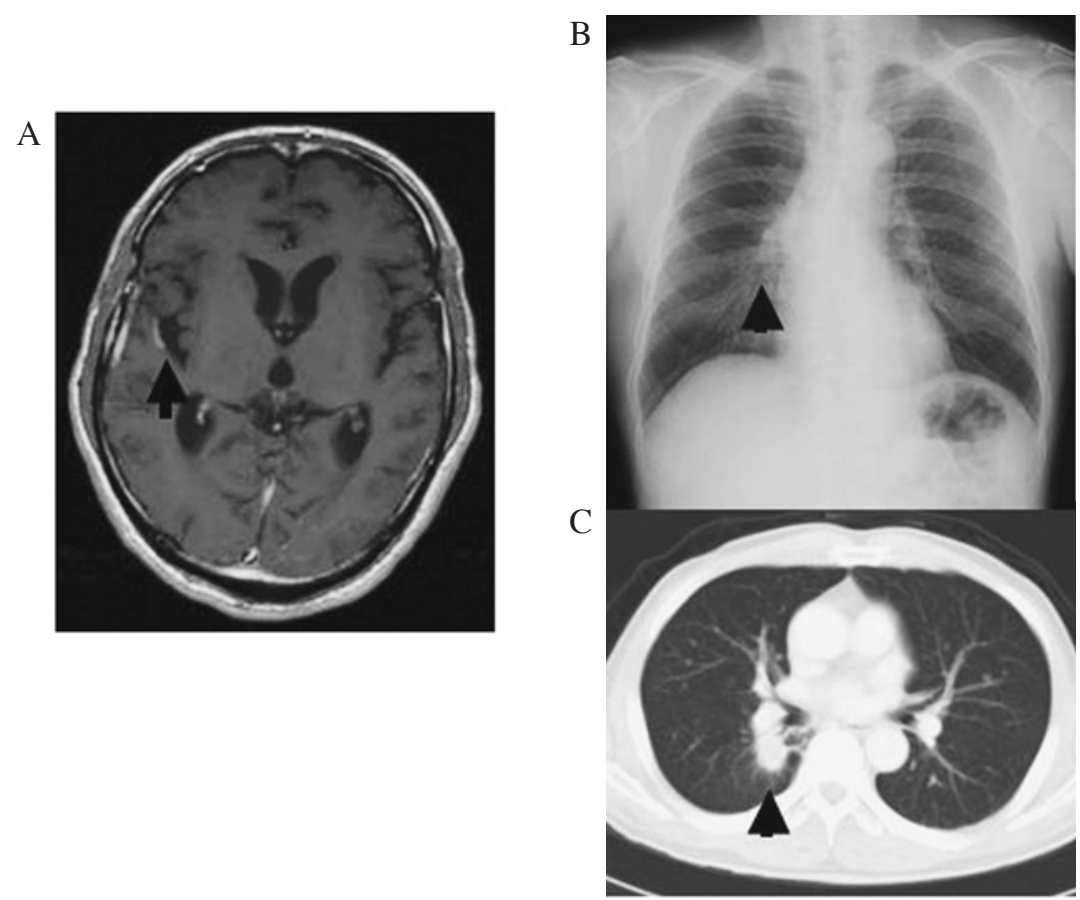

Fig. 1. Images taken before gefitinib treatment.

A: Brain gadolinium-enhanced T1-weighted image. High-intensity signals in the opercular parts of the temporal lobe are shown. B: Chest X-ray. A nodular shadow at a hilus in the right lung is indicated. C: CT scan. A nodular shadow with spicule formation and hilar lymph node swelling are shown. 
using an Ommaya reservoir was used to control the patient's intracranial pressure on 1 November 2006.

Conventional cytotoxic chemotherapy was contraindicated because of the patient's poor performance status, and we predicted that such treatment would not be effective against carcinomatous meningitis. Therefore, treatment with gefitinib ( $250 \mathrm{mg} /$ day) was initiated on 7 November 2006. WBI (36 Gray total) was added to the regimen 16 days later. The patient's primary tumor regressed quickly after gefitinib treatment, and the combination of gefitinib and WBI gradually eased the patient's headache and decreased his intracranial pressure. Cytological examination of the patient's CSF revealed the presence of Class II cells that were unable to recognize carcinoma cells, and the Ommaya reservoir was removed on 18 November 2006. The patient was discharged on 17 December 2006 and has since continued receiving gefitinib as an outpatient. A chest CT scan on 20 February 2007 (Fig. 2A) showed that the patient exhibited partial response as assessed by the Response Evaluation Criteria in Solid Tumors guidelines. The only adverse effect of the therapy was low-grade diarrhea, and no drug-induced lung disease was observed. The patient has maintained good performance status, and the regressed size of the primary lung tumor has been maintained for more than 10 months (Fig. 2B, chest CT scan on 18 September 2007). The high-intensity signals were largely diminished in gadolinium-enhanced T1weighted images collected on 16 November 2007 (Fig. 2C).

On 17 November 2006, 10 days after beginning gefitinib treatment, the concentrations of gefitinib in the patient's serum and CSF were determined at two time points, by Shin Nippon Biomedical Laboratories, Pharmaco-kinetics, and Bioanalysis Center (Wakayama), using a previously described method (Jones et al. 2002). It is notable that the level of gefitinib in the patient's CSF was roughly $0.9 \mathrm{nM}$ both before administration of the drug in the morning and at $2 \mathrm{hrs}$ after administration, whereas the gefitinib level in the patient's serum was $117 \mathrm{nM}$ before morning administration of the drug and $132 \mathrm{nM}$ at $2 \mathrm{hrs}$ after administration (Table 1).

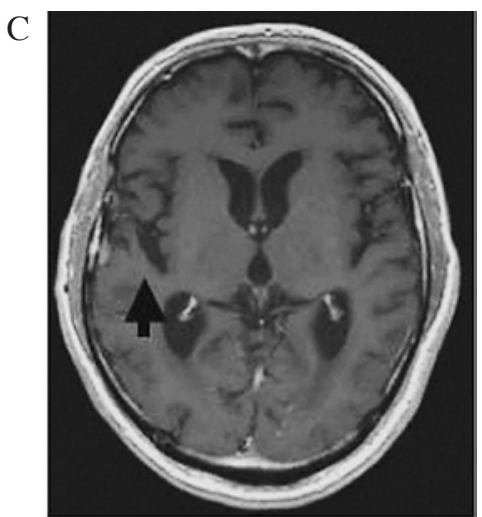

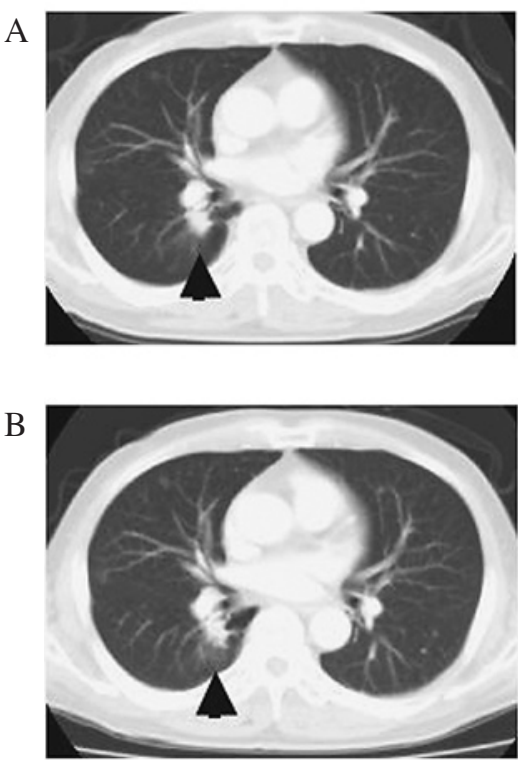

Fig. 2. Images taken during gefitinib treatment.

A: Chest CT scan on 20 February 2007. The nodular shadow had regressed. B: CT scan on 18 September 2007. The regressed size of the nodular shadow was maintained. C: Brain gadoliniumenhanced T1-weighted image taken on 16 November 2007. High-intensity signals have largely diminished. 
TABLE 1.

\begin{tabular}{lccc}
\hline Time of sample collection & Serum & CSF & (nM) \\
\hline Before morning administration & 117 & 0.9 & \\
Two hours after & 132 & 0.9 & \\
\hline
\end{tabular}

\section{DiscuSsion}

Cases of carcinomatous meningitis with NSCLC are on the rise because patients with NSCLC are surviving longer now than ever before. Carcinomatous meningitis is currently treated by radiotherapy, systemic chemotherapy, and intrathecal chemotherapy; however, no effective drug for NSCLC has been approved as safe for intrathecal injection. Although radiotherapy is common, its therapeutic effects are limited. Thus, the prognosis of patients with carcinomatous meningitis is poor.

The response of NSCLC patients to gefitinib is highly associated with mutations in the EGF kinase domain. Two major hotspots have been reported in $E G F$, in-frame deletions at codons 746 to 750 (E746 to A750) in exon 19 and an amino acid substitution at codon 858 (L858R) in exon 21. Both retrospective and prospective studies report a $70-80 \%$ response rate in NSCLC patients harboring an EGF mutation (Inoue et al. 2006). In our patient, a deletion in exon 19 was detected in the primary lung tumor samples, although cytological examination of the patient's CSF did not detect the EGF mutation, possibly because of the low number of cancer cells in the fluid. The primary tumor in this case responded quickly to gefitinib treatment, and the patient's carcinomatous meningitis symptoms and cytological status improved following gefitinib treatment combined with radiotherapy.

The effects of gefitinib on metastatic lesions in the central nervous system have been reported (Namba et al. 2004; Kim et al. 2005; Sakai et al. 2006; Wu et al. 2007). The response rate in patients with brain metastases is reportedly similar to that in patients with NSCLC (Wu et al. 2007). On the other hand, only case reports of carcinomatous meningitis have been published, probably because of the small number of carcino- matous meningitis cases treated with gefitinib (Namba et al. 2004; Kim et al. 2005; Sakai et al. 2006). Although radiotherapy alone produced only scant improvement in our patient's symptoms, he has been able to control his condition for more than 6 months. Thus, we conclude that gefitinib was effective against carcinomatous meningitis in our case.

The concentration of gefitinib in the patient's CSF $(0.9 \mathrm{nM})$ was less than $1 \%$ of the serum concentration (117 and $132 \mathrm{nM}$ ). The low concentration of gefitinib in the CSF may be attributable to its low permeability at the blood-brain barrier, which affects the delivery of many drugs. It was previously demonstrated that the concentration of $\left[{ }^{14} \mathrm{C}\right]$-labeled gefitinib in normal rat brain and spinal cord was only $2 \%$ of that in lung, suggesting that gefitinib does not readily cross the bloodbrain barrier (McKillop et al. 2004); however, the concentration of gefitinib in the CSF was not measured. Only one previous report considered the concentration of gefitinib in the CSF of NSCLC patients with leptomeningeal metastases (Jackman et al. 2006). In that study, increasing doses of gefitinib resulted in increasing concentrations of gefitinib in the CSF, with the concentration of gefitinib in the patients' CSF varying from 6.2 to $18 \mathrm{nM}$ following a 500-mg dose (double the dose in our case) and reaching $42 \mathrm{nM}$ following a 1,000-mg dose. Given that regimen, the patients' carcinomatous meningitis was controlled for several months. A second study reported that the $\mathrm{IC}_{50}$ of gefitinib was $10-20 \mathrm{nM}$ (higher than the CSF concentration in our case) in $E G F$-mutant cells and $4 \mu \mathrm{M}$ in cells harboring wild-type $E G F$ (Mukohara et al. 2005). Thus, a higher dose of gefitinib may be indicated in patients with carcinomatous meningitis.

Gefitinib should be considered as a treatment option for NSCLC patients with carcinomatous meningitis when the tumor harbors an EGF mutation. Moreover, in cases of mutant $E G F$-related lung cancer with brain metastasis or carcinomatous meningitis that cannot be controlled by the standard dose of gefitinib, a higher dose of gefitinib should be utilized. 


\section{References}

Inoue, A., Suzuki, T., Fukuhara, T., Maemondo, M., Kimura, Y., Morikawa, N., Watanabe, H., Saijo, Y. \& Nukiwa, T. (2006) Prospective phase II study of gefitinib for chemotherapynaive patients with advanced non-small-cell lung cancer with epidermal growth factor receptor gene mutations. $J$. Clin. Oncol., 24, 3340-3346.

Jackman, D.M., Holmes, A.J., Lindeman, N., Wen, P.Y., Kesari, S., Borras, A.M., Bailey, C., de Jong, F., Jänne, P.A. \& Johnson, B.E. (2006) Response and resistance in a nonsmall cell lung cancer patient with an epidermal growth factor receptor mutation and leptomeningeal metastases treated with high-dose gefitinib. J. Clin. Oncol., 24, 4517-4520.

Jones, H.K., Stafford, L.E., Swaisland, H.C. \& Payne, R. (2002) A sensitive assay for ZD1839 (Iressa) in human plasma by liquid-liquid extraction and high performance liquid chromatography with mass spectrometric detection: validation and use in Phase I clinical trials. J. Pharm. Biomed. Anal., 29, 221-228.

Kim, M.K., Lee, K.H., Lee, J.K., Choi, J.H. \& Hyun, M.S. (2005) Gefitinib is also active for carcinomatous meningitis in NSCLC. Lung Cancer, 50, 265-269.

McKillop, D., Hutchison, M., Partridge, E.A., Bushby, N., Cooper, C.M., Clarkson-Jones, J.A., Herron, W. \& Swaisland, H.C. (2004) Metabolic disposition of gefitinib, an epidermal growth factor receptor tyrosine kinase inhibitor, in rat, dog and man. Xenobiotica, 34, 917-934.
Mukohara, T., Engelman, J.A., Hanna, N.H., Yeap, B.Y., Kobayashi, S., Lindeman, N., Halmos, B., Pearlberg, J., Tsuchihashi, Z., Cantley, L.C., Tenen, D.G., Johnson, B.E. \& Jänne, P.A. (2005) Differential effects of gefitinib and cetuximab on non-small cell lung cancers bearing epidermal growth factor receptor mutations. J. Natl. Cancer Inst., 97, 1185-1194.

Nagai, Y., Miyazawa, H., Huqun, T., Tanaka, T., Udagawa, K., Kato, M., Fukuyama, S., Yokote, A., Kobayashi, K., Kanazawa, M. \& Hagiwara, K. (2005) Genetic heterogeneity of the epidermal growth factor receptor in non-small cell lung cancer cell lines revealed by a rapid and sensitive detection system, the peptide nucleic acid-locked nucleic acid PCR clamp. Cancer Res., 65, 7276-7282.

Namba, Y., Kijima, T., Yokota, S., Niinaka, M., Kawamura, S., Iwasaki, T., Takeda, Y., Kimura, H., Okada, T., Yamaguchi, T., Nakagawa, M., Okumura, Y., Maeda, H. \& Ito, M. (2004) Gefitinib in patients with brain metastases from non-small cell lung cancer: review of 15 clinical cases. Clin. Lung Cancer, 6, 123-128.

Sakai, M., Ishikawa, S., Ito, H., Ozawa, Y., Yamamoto, T., Yamamoto, T., Onizuka, M. \& Sakakibara, Y. (2006) Carcinomatous meningitis from non-small cell lung cancer responding to gefitinib. Int. J. Clin. Oncol., 11, 243-245.

Wu, C., Li, Y.L., Wang, Z.M., Li, Z., Zhang, T.X. \& Wei, Z. (2007) Gefitinib as palliative therapy for lung adenocarcinoma metastatic to the brain. Lung Cancer, 57, 359-364. 